\title{
ON THE AXIOMATIZATION OF CONVEX SUBSETS OF BANACH SPACES
}

\author{
VALERIO CAPRARO AND TOBIAS FRITZ
}

(Communicated by Thomas Schlumprecht)

\begin{abstract}
We prove that any convex-like structure in the sense of Nate Brown is affinely and isometrically isomorphic to a closed convex subset of a Banach space. This answers an open question of Brown. As an intermediate step, we identify Brown's algebraic axioms as equivalent to certain well-known axioms of abstract convexity. We conclude with a new characterization of convex subsets of Banach spaces.
\end{abstract}

\section{INTRODUCTION}

While studying an invariant of $\mathrm{II}_{1}$-factors related to Connes' embedding conjecture, Brown $[\mathrm{Br}$ found that there is a natural way of defining convex combinations on this invariant. However, there seemed to be no evident embedding of this set into some linear space such that the convex combinations are precisely those inherited from the vector space structure. Searching for an axiomatization of those metric spaces where it makes sense to talk about convex combinations without having any linear structure, he proposed the notion of a convex-like structure. The obvious examples of convex-like structures are closed convex subsets of Banach spaces. The very basic question is whether any convex-like structure is of this form. Besides being interesting in itself, this question also has a technical reason: there are many properties of convex combinations which are trivial to verify in vector spaces, but are hard to prove in the context of convex-like structures. Here we give a positive answer to this problem.

Actually, we obtain this result as a consequence of a more general one: four of the five Brown's axioms, exactly the algebraic ones, are equivalent to certain wellknown axioms of abstract convexity. These were introduced by Stone [St] and have since been discussed and sometimes rediscovered, modulo minor variations, several times ([Fr], $[\mathrm{Gu},[\mathrm{Mo},[\mathrm{PR}],[\mathrm{Se}])$ using various terminology; here, we shall follow the notation and terminology of $[\mathrm{Fr}$.

Received by the editors May 27, 2011 and, in revised form, September 26, 2011.

2010 Mathematics Subject Classification. Primary 52A01; Secondary 46L10.

Key words and phrases. Convex-like structure, Stone's barycentric calculus, convex space.

The first author was supported by Swiss SNF Sinergia project CRSI22-130435.

The second author was supported by the EU STREP QCS. 


\section{Convex-Like structures and CONVEx spaces}

In order to be precise, and also for the convenience of the reader, we recall the definitions and the Stone embedding theorem which we are going to use. The following two definitions are both abstractions of the properties of convex combinations in vector spaces.

Definition $1([\mathrm{Br}])$. Let $(X, d)$ be a complete metric space. Take $X^{(n)}=X \times \cdots \times$ $X$ to be the $n$-fold Cartesian product and $\operatorname{Prob}_{n}$ the set of probability measures on the $n$-element set $\{1,2, \ldots, n\}$ endowed with the $\ell_{1}$-metric $\|\mu-\tilde{\mu}\|=\sum_{i=1}^{n} \mid \mu(i)-$ $\tilde{\mu}(i) \mid$. We say that $(X, d)$ has a convex-like structure if for every $n \in \mathbb{N}$ and $\mu \in$ $\operatorname{Prob}_{n}$ there is given a continuous map $\gamma_{\mu}: X^{(n)} \rightarrow X$ such that

$(\gamma .1)$ for each permutation $\sigma \in S_{n}$ and $x_{1}, \ldots, x_{n} \in X$,

$$
\gamma_{\mu}\left(x_{1}, \ldots, x_{n}\right)=\gamma_{\mu \circ \sigma}\left(x_{\sigma(1)}, \ldots, x_{\sigma(n)}\right) \text {; }
$$

$(\gamma .2)$ if $x_{1}=x_{2}$, then $\gamma_{\mu}\left(x_{1}, x_{2}, \ldots, x_{n}\right)=\gamma_{\tilde{\mu}}\left(x_{1}, x_{3}, \ldots, x_{n}\right)$, where $\tilde{\mu} \in \operatorname{Prob}_{n-1}$ is given by $\tilde{\mu}(1)=\mu(1)+\mu(2)$ and $\tilde{\mu}(j)=\mu(j+1)$ for $2 \leq j \leq n-1$;

$(\gamma .3)$ if $\mu(i)=1$, then $\gamma_{\mu}\left(x_{1}, \ldots, x_{n}\right)=x_{i}$;

$(\gamma .4)$ the metric compatibility axiom 1 holds for all $y_{1}, \ldots, y_{n} \in X$,

$$
d\left(\gamma_{\mu}\left(x_{1}, \ldots, x_{n}\right), \gamma_{\mu}\left(y_{1}, \ldots, y_{n}\right)\right) \leq \sum_{i=1}^{n} \mu(i) d\left(x_{i}, y_{i}\right) ;
$$

$(\gamma .5)$ for all $\nu \in \operatorname{Prob}_{2}, \mu \in \operatorname{Prob}_{n}, \tilde{\mu} \in \operatorname{Prob}_{m}$ and $x_{1}, \ldots, x_{n}, \tilde{x}_{1}, \ldots, \tilde{x}_{m} \in X$,

$$
\gamma_{\nu}\left(\gamma_{\mu}\left(x_{1}, \ldots, x_{n}\right), \gamma_{\tilde{\mu}}\left(\tilde{x}_{1}, \ldots, \tilde{x}_{m}\right)\right)=\gamma_{\eta}\left(x_{1}, \ldots, x_{n}, \tilde{x}_{1}, \ldots, \tilde{x}_{m}\right),
$$

where $\eta \in \operatorname{Prob}_{n+m}$ is given by $\eta(i)=\nu(1) \mu(i)$, if $1 \leq i \leq n$, and $\eta(j+n)=$ $\nu(2) \tilde{\mu}(j)$, if $1 \leq j \leq m$.

The idea behind this definition is that the $n$-ary operation $\gamma_{\mu}$ is supposed to stand for a convex combination with weights given by the coefficients of $\mu$ :

$$
\gamma_{\mu}\left(x_{1}, \ldots, x_{n}\right) \widehat{=} \sum_{i=1}^{n} \mu(i) x_{i} .
$$

With this intuition, it is clear why one wants the properties $(\gamma .1)$ through $(\gamma .5)$ to hold.

Definition $2([\mathrm{Fr}])$. A convex space is given by a set $X$ and a family of binary operations $\left\{c c_{\lambda}\right\}_{\lambda \in[0,1]}$ on $X$ such that

(cs.1) $c c_{0}(x, y)=x \quad \forall x, y \in X$,

(cs.2) $c c_{\lambda}(x, x)=x \quad \forall x \in X, \lambda \in[0,1]$,

(cs.3) $c c_{\lambda}(x, y)=c c_{1-\lambda}(y, x) \quad \forall x, y \in X, \lambda \in[0,1]$,

(cs.4) $c c_{\lambda}\left(c c_{\mu}(x, y), z\right)=c c_{\lambda \mu}\left(x, c c_{\nu}(y, z)\right) \quad \forall x, y, z \in X, \lambda, \mu \in[0,1]$, where $\nu$ is arbitrary if $\lambda=\mu=1$ and $\nu=\frac{\lambda(1-\mu)}{1-\lambda \mu}$ otherwise.

Now an interpretation analogous to (11) holds: the $c c_{\lambda}$ simply model binary convex combinations with weight $\lambda$ :

$$
c c_{\lambda}(x, y) \widehat{=} \lambda x+(1-\lambda) y .
$$

\footnotetext{
${ }^{1}$ Brown's original metric compatibility axiom actually consisted of two conditions. See Remark 11
} 
Again, properties (cs.1) through (cs.4) clearly hold for convex combinations in vector spaces.

Our first result follows now. It states that convex-like structures differ from convex spaces just by the metric compatibility axiom $(\gamma \cdot 4)$. The following equation (3) is motivated by the correspondences (11) and (2).

A piece of notation: when $\mu(1)=\lambda \in[0,1]$ and $\mu(2)=1-\lambda$ are the parameters of a distribution $\mu \in \mathrm{Prob}_{2}$, then we also write $\gamma_{\lambda, 1-\lambda}$ instead of $\gamma_{\mu}$.

Theorem 3. The algebraic axioms $(\gamma .1),(\gamma .2),(\gamma .3)$ and $(\gamma .5)$ of Definition 1 are equivalent to the axioms of a convex space in Definition 2. More precisely: for a given set $X$, a convex-like structure on $X$ and the structure of a convex space on $X$ mutually determine each other by the identity

$$
c c_{\lambda}(x, y)=\gamma_{\lambda, 1-\lambda}(x, y) .
$$

Proof. Let us start by proving that Brown's axioms $(\gamma .1),(\gamma .2),(\gamma .3)$ and $(\gamma .5)$ for a convex-like structure imply the axioms of convex spaces when the $c c_{\lambda}$ are defined as in (3).

\begin{tabular}{l|l|l|}
\hline$(\operatorname{cs} .1)$ & We have $c c_{0}(x, y)=\gamma_{0,1}(x, y)=y$ thanks to Brown's axiom $(\gamma .3)$ \\
\cline { 1 - 1 }$(\operatorname{cs} .2)$ & We have $c c_{\lambda}(x, x)=\gamma_{\lambda, 1-\lambda}(x, x)$ thanks to Brown's axiom $(\gamma .2)$ \\
\cline { 1 - 1 }$(\operatorname{cs} .3)$ & We have
\end{tabular}

$$
c c_{\lambda}(x, y)=\gamma_{\lambda, 1-\lambda}(x, y)=\gamma_{1-\lambda, \lambda}(y, x)=c c_{1-\lambda}(y, x)
$$

thanks to Brown's axiom $(\gamma \cdot 1)$.

(cs.4) This is implied by the previous axioms when $\lambda=\mu=1$, so it is enough to treat the case $\lambda \mu \neq 1$. We will evaluate $c c_{\lambda}\left(c c_{\mu}(x, y), z\right)$ and $c c_{\lambda \mu}(x$, $\left.c c_{\frac{\lambda(1-\mu)}{1-\lambda \mu}}(y, z)\right)$ separately and obtain two identical expressions. Using ax$\operatorname{iom}(\gamma .5)$, we have

$$
c c_{\lambda}\left(c c_{\mu}(x, y), z\right)=\gamma_{\eta}(x, y, z),
$$

where $\eta(1)=\lambda \mu, \eta(2)=\lambda(1-\mu)$ and $\eta(3)=1-\lambda$. On the other hand, the same $(\gamma .5)$ also implies that

$$
c c_{\lambda \mu}\left(x, c c_{\frac{\lambda(1-\mu)}{1-\lambda \mu}}(y, z)\right)=\gamma_{\eta}(x, y, z)
$$

with the same distribution $\eta \in \mathrm{Prob}_{3}$.

We now proceed to the proof of the converse implication. Given a family of binary operations $c c_{\lambda}$ which satisfy the axioms of convex spaces, we first define $\gamma_{\lambda, 1-\lambda}$ according to equation (3). Given this, it then has to be shown that there exist unique choices for the $\gamma_{\eta}$ with $\eta \in \operatorname{Prob}_{n}$ for all $n \in \mathbb{N}$ such that $(\gamma .1),(\gamma .2),(\gamma .3)$ and $(\gamma .5)$ hold. Since $\gamma_{\iota}=\operatorname{id}_{X}$ for $\iota \in \operatorname{Prob}_{1}$, and, for $n \geq 3$, any $\eta \in \operatorname{Prob}_{n}$ can appear on the right-hand side of $(\gamma .5)$, we can already conclude the uniqueness: it is enough to specify the $\gamma_{\eta}$ with $\eta \in \operatorname{Prob}_{n}$ for $n=2$.

We still need to show the existence part. To this end, we first define $\gamma_{\mu}$ for $\mu \in \operatorname{Prob}_{n}$ recursively by setting

$$
\gamma_{\mu}\left(x_{1}, \ldots, x_{n}\right) \equiv\left\{\begin{array}{cc}
x_{n} & \text { if } \mu(n)=1, \\
c c_{1-\mu(n)}\left(\gamma_{\nu}\left(x_{1}, \ldots, x_{n-1}\right), x_{n}\right) & \text { if } \mu(n) \neq 1,
\end{array}\right.
$$

where $\nu \in \operatorname{Prob}_{n-1}$ is given by $\nu(i)=\frac{\mu(i)}{1-\mu(n)}$. So one obtains all $n$-ary operations by repeated applications of the binary ones. 
Due to $c c_{\lambda}(x, y)=c c_{1-\lambda}(y, x)$, this definition respects the permutation invariance $(\gamma .1)$ when the permutation does nothing but exchange $x_{1}$ with $x_{2}$. For any $n \geq 3$, the definition can be expanded to

$\gamma_{\mu}\left(x_{1}, \ldots, x_{n}\right)=\left\{\begin{array}{cc}x_{n} & \text { if } \mu(n)=1 \\ x_{n-1} & \text { if } \mu(n-1)=1 \\ c c_{1-\mu(n)}\left(c c_{1-\frac{\mu(n-1)}{1-\mu(n)}}\left(\gamma_{\eta}\left(x_{1}, \ldots, x_{n-2}\right), x_{n-1}\right), x_{n}\right) \text { otherwise }\end{array}\right.$

with $\eta \in \operatorname{Prob}_{n-2}$ given by $\eta(i)=\frac{\nu(i)}{1-\nu(n-1)}=\frac{\mu(i)}{1-\mu(n-1)-\mu(n)}$. Writing $y=$ $\gamma_{\eta}\left(x_{1}, \ldots, x_{n-2}\right)$, the associativity rule (cs.4) gives

$$
\begin{aligned}
\gamma_{\mu}\left(x_{1}, \ldots, x_{n}\right) & =c c_{1-\mu(n)}\left(c c_{1-\frac{\mu(n-1)}{1-\mu(n)}}\left(y, x_{n-1}\right), x_{n-2}\right) \\
& =c c_{1-\mu(n-1)-\mu(n)}\left(y, c c_{\frac{\mu(n-1)}{\mu(n-1)+\mu(n)}}\left(x_{n-1}, x_{n}\right)\right)
\end{aligned}
$$

and hence (cs.3) implies the permutation invariance $(\gamma .1)$ also for $\gamma_{\mu}$ when exchanging $x_{n-1}$ with $x_{n}$ while keeping all other arguments fixed. By the recursive definition of $\gamma_{\mu}$, this argument also proves invariance under transposing $x_{k-1}$ with $x_{k}$ for any $k<n$. Hence now we know that $(\gamma .1)$ holds with respect to all transpositions of neighboring arguments. But since the latter generate all permutations, $(\gamma .1)$ holds in complete generality.

With this, Brown's $(\gamma .2)$ and $(\gamma .3)$ are straightforward to prove: by $(\gamma .1)$, the property $(\gamma .2)$ is equivalent to the analogous one with $x_{n-1}=x_{n}$ instead of $x_{1}=$ $x_{2}$. The latter follows from the previous considerations together with the axiom $c c_{\lambda}(x, x)=x$. The statement $(\gamma \cdot 3)$, for $i=1$, follows directly from the definition of $\gamma_{\mu}$ together with $c c_{1}(x, y)=y$.

Finally, we prove $(\gamma .5)$ by induction on $m$. For $m=1$, this equation coincides with our definition of its right-hand side. For $m \geq 2$, we can assume $\tilde{\mu}(m) \neq 1$ by appealing to $(\gamma .1)$. Then the left-hand side of $(\gamma .5)$ can be written as

$$
c c_{\nu(1)}\left(\gamma_{\mu}\left(x_{1}, \ldots, x_{n}\right), c c_{1-\tilde{\mu}(m)}\left(\gamma_{\mu^{\prime}}\left(\tilde{x}_{1}, \ldots, \tilde{x}_{m-1}\right), \tilde{x}_{m}\right)\right),
$$

where $\mu^{\prime}(i)=\frac{\tilde{\mu}(i)}{1-\tilde{\mu}(m)}$. An application of the associativity rule (cs.4) evaluates this to

$$
c c_{1-\tilde{\mu}(m) \nu(2)}\left(c c_{\frac{\nu(1)}{1-\tilde{\mu}(m) \nu(2)}}\left(\gamma_{\mu}\left(x_{1}, \ldots, x_{n}\right), \gamma_{\mu^{\prime}}\left(\tilde{x}_{1}, \ldots, \tilde{x}_{m-1}\right)\right), \tilde{x}_{m}\right) .
$$

Now by the induction assumption, this can be written as

$$
c c_{1-\tilde{\mu}(m) \nu(2)}\left(\gamma_{\delta}\left(x_{1}, \ldots, x_{n}, \tilde{x}_{1}, \ldots, \tilde{x}_{m-1}\right), \tilde{x}_{m}\right),
$$

where $\delta$ is the distribution with $\delta(i)=\frac{\nu(1)}{1-\tilde{\mu}(m) \nu(2)} \mu(i)$ for $1 \leq i \leq n$ and $\delta(i+n)=$ $\frac{\nu(2)}{1-\tilde{\mu}(m) \nu(2)} \tilde{\mu}(i)$. This equation is the definition of the right-hand side of $(\gamma .5)$.

\section{Embeddings into VECTOR SPACES}

The following theorem and proof have been adapted from [St].

Theorem 4 ([St]). A convex space embeds into a real vector space with (2) if and only if the following cancellation property holds:

$$
c c_{\lambda}(x, y)=c c_{\lambda}(x, z) \text { with } \lambda \in(0,1) \quad \Longrightarrow \quad y=z \text {. }
$$


Proof. It is clear that every convex subset of a vector space satisfies this cancellation property, so it remains to prove the "if" direction.

Given a convex space $X$ with the cancellation property, we define a real vector space as follows: let $V_{X}$ be the real vector space formally generated by all points of $X$, so that $V(X)$ has a basis $\left(e_{x}\right)_{x \in X}$. The vectors of the form

$$
e_{c c_{\lambda}(x, y)}-\lambda e_{x}-(1-\lambda) e_{y}, \quad x, y \in X, \lambda \in[0,1],
$$

generate a subspace $U_{X} \subseteq V_{X}$. Let $W_{X}$ be the quotient space $V_{X} / U_{X}$ and let $\tilde{e}_{x}$ denote the image of $e_{x}$ under the canonical projection. Then the mapping

$$
X \rightarrow W_{X}, \quad x \mapsto \tilde{e}_{x}
$$

preserves convex combinations.

In order to see that this mapping is injective, it is first necessary to take a closer look at the subspace $U_{X}$. The vectors in $U_{X}$ are all the finite linear combinations of vectors of the form (44). Taking the coefficients $\alpha_{i}$ and $\beta_{i}$ to be nonnegative, we can write such a linear combination as

$$
\sum_{i=1}^{m} \alpha_{i}\left(e_{c c_{\lambda_{i}}\left(a_{i}, b_{i}\right)}-\lambda_{i} e_{a_{i}}-\left(1-\lambda_{i}\right) e_{b_{i}}\right)-\sum_{i=1}^{m} \beta_{i}\left(e_{c c_{\mu_{i}}\left(c_{i}, d_{i}\right)}-\mu_{i} e_{c_{i}}-\left(1-\mu_{i}\right) e_{d_{i}}\right)
$$

for certain points $a_{i}, b_{i}, c_{i}, d_{i} \in X$ and weights $\lambda_{i}, \mu_{i} \in[0,1]$. We split this into positive terms and negative terms as follows:

$$
\begin{aligned}
\sum_{i=1}^{m}\left(\alpha_{i} e_{c c_{\lambda_{i}}\left(a_{i}, b_{i}\right)}+\beta_{i} \mu_{i} e_{c_{i}}\right. & \left.+\beta_{i}\left(1-\mu_{i}\right) e_{d_{i}}\right) \\
& -\sum_{i=1}^{m}\left(\beta_{i} e_{c c_{\mu_{i}}\left(c_{i}, d_{i}\right)}+\alpha_{i} \lambda_{i} e_{a_{i}}+\alpha_{i}\left(1-\lambda_{i}\right) e_{b_{i}}\right) .
\end{aligned}
$$

This expression has two important properties: firstly, the sum of the coefficients of all negative terms equals the sum of the coefficients of all positive terms, namely $\sum_{i}\left(\alpha_{i}+\beta_{i}\right)$. If we assume this sum to be 1 without loss of generality, then, secondly, both sums are just convex combinations. Interpreting these as convex combinations in $X$, these sums moreover define the same point in $X$.

We now prove the required injectivity property by showing that $\tilde{e}_{x}=\tilde{e}_{y}$ implies $x=y$ for any two points $x, y \in X$. The equation $\tilde{e}_{x}=\tilde{e}_{y}$ holds whenever $e_{x}-e_{y}$ lies in $U_{X}$. If this is the case, then there exists an expression of the form (5) where the first sum contains the term $\kappa e_{x}$ for some $\kappa>0$ and the second sum contains the term $\kappa e_{y}$ for the same $\kappa$, while all other terms cancel. Then by the above, the two sums in (5) define convex combinations of the same points with the same weights, except that the first one contains the point $x$ with weight $\kappa$, while the second one contains the point $y$ with weight $\kappa$. If one combines all the other points besides these $x$ and $y$ to a single point $z$ which carries a weight $1-\kappa$, one ends up with the equation

$$
c c_{\kappa}(x, z)=c c_{\kappa}(y, z),
$$

which implies $x=y$ by the cancellation condition.

The similarity to the Grothendieck construction which embeds a cancellative abelian monoid into an abelian group should be clear. Just like the latter proceeds by constructing a left adjoint to the inclusion functor of the category of abelian groups into the category of abelian monoids, Stone's embedding theorem implicitly 
constructs a left adjoint to the inclusion functor of the category of real vector spaces into the category of convex spaces.

We will soon prove that the metric compatibility axiom $(\gamma .4)$ guarantees that the cancellation condition holds in a convex-like structure. This requires a bit of preparation:

Lemma 5. If the equation

$$
c c_{\lambda}(y, x)=c c_{\lambda}(z, x)
$$

holds for some $x, y, z \in X$ and $\lambda \in(0,1)$, then it also holds for all $\lambda \in(0,1)$.

Proof. Let us write $\lambda_{0}$ for the original value for which the equation holds. Then for all $\lambda<\lambda_{0}$,

$$
c c_{\lambda}(y, x)=c c_{\lambda / \lambda_{0}}\left(c c_{\lambda_{0}}(y, x), x\right)=c c_{\lambda / \lambda_{0}}\left(c c_{\lambda_{0}}(z, x), x\right)=c c_{\lambda}(z, x)
$$

by (cs.4) and (cs.2), so that the equation is also true in that case. Hence it is enough to find a sequence $\left(\lambda_{n}\right)_{n \in \mathbb{N}}$ with $\lambda_{n} \stackrel{n \rightarrow \infty}{\longrightarrow} 1$ for which the equation holds. We construct such a sequence by defining $\lambda_{n+1}=\frac{2 \lambda_{n}}{1+\lambda_{n}}$, for which an inductive argument shows the validity of the equation:

$$
\begin{aligned}
c c_{\lambda_{n+1}}(y, x) & =c c_{\lambda_{n} /\left(1+\lambda_{n}\right)}\left(y, c c_{\lambda_{n}}(y, x)\right)=c c_{\lambda_{n} /\left(1+\lambda_{n}\right)}\left(y, c c_{\lambda_{n}}(z, x)\right) \\
& =c c_{\lambda_{n} /\left(1+\lambda_{n}\right)}\left(z, c c_{\lambda_{n}}(y, x)\right)=c c_{\lambda_{n} /\left(1+\lambda_{n}\right)}\left(z, c c_{\lambda_{n}}(z, x)\right)=c c_{\lambda_{n+1}}(z, x) .
\end{aligned}
$$

Corollary 6. Let $X$ be a convex-like structure. Then there is a linear embedding of $X$ into some vector space.

Proof. By Theorem 3 and Theorem 4 it suffices to prove the cancellation property: if $\gamma_{\lambda, 1-\lambda}(x, y)=\gamma_{\lambda, 1-\lambda}(x, z)$ for some $\lambda \in(0,1)$, then $y=z$. By the previous lemma, we know that if $\gamma_{\lambda, 1-\lambda}(x, y)=\gamma_{\lambda, 1-\lambda}(x, z)$ holds for some $\lambda \in(0,1)$, then it holds for all $\lambda \in(0,1)$. But then we get from $(\gamma .4)$, for any $\lambda>0$,

$$
\begin{aligned}
d(y, z) & \leq d\left(y, \gamma_{\lambda, 1-\lambda}(x, y)\right)+d\left(z, \gamma_{\lambda, 1-\lambda}(x, z)\right) \\
& \leq \lambda d(x, y)+\lambda d(x, z)=\lambda[d(x, y)+d(x, z)] .
\end{aligned}
$$

Since $\lambda$ was arbitrary, we conclude that $d(y, z)=0$, and hence $y=z$.

Remark 7. The proof of Corollary 6 indeed strongly depends on Brown's axiom $(\gamma .4)$ in $[\mathrm{Fr}$ there are examples of convex spaces which do not embed into a vector space.

\section{ISOMETRIC EMBEDDINGS INTO NORMED SPACES}

Lemma 8. Let $(X, d)$ be a metric space which is a convex subset $X \subseteq E$ of some vector space $E$ such that

$$
d(\lambda y+(1-\lambda) x, \lambda z+(1-\lambda) x) \leq \lambda d(y, z) \quad \forall x, y \in X, \lambda \in[0,1]
$$

holds. Then there is a norm $\|\cdot\|$ on $E$ such that for all $x, y \in X$,

$$
d(x, y)=\|x-y\| .
$$




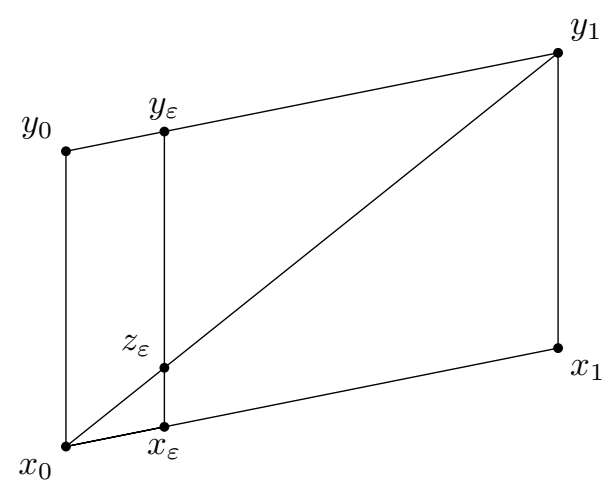

Figure 1. Illustration of the proof of Lemma 8 .

Proof. As a special case, (6) gives for $z=x$,

$$
d(\lambda y+(1-\lambda) x, x) \leq \lambda d(y, x)
$$

which yields, in combination with the triangle inequality,

$$
d(y, x) \leq d(y, \lambda y+(1-\lambda) x)+d(\lambda y+(1-\lambda) x, x) \leq(1-\lambda) d(y, x)+\lambda d(y, x) .
$$

Since the term on the left-hand side equals the term on the right-hand side, we deduce that both inequalities are actually equalities. In particular, the metric is "uniform on lines" in the sense that

$$
d(x,(1-\lambda) x+\lambda y)=\lambda d(x, y) \quad \forall x, y \in X, \lambda \in[0,1] .
$$

Now in order to prove the assertion, it needs to be shown that $d$ is translationinvariant in the following sense: suppose that $x_{0}, x_{1}, y_{0}, y_{1} \in X$ are such that

$$
y_{1}-x_{1}=y_{0}-x_{0}
$$

then $d\left(x_{1}, y_{1}\right)=d\left(x_{0}, y_{0}\right)$. See Figure 1 for an illustration. For $\varepsilon \in(0,1)$, we will also consider the points

$x_{\varepsilon}=\varepsilon x_{1}+(1-\varepsilon) x_{0}, \quad y_{\varepsilon}=\varepsilon y_{1}+(1-\varepsilon) y_{0}, \quad z_{\varepsilon}=(1-\varepsilon) x_{\varepsilon}+\varepsilon y_{\varepsilon}=\varepsilon y_{1}+(1-\varepsilon) x_{0}$.

Then by the assumption (6),

$$
d\left(x_{\varepsilon}, z_{\varepsilon}\right)=d\left(\varepsilon x_{1}+(1-\varepsilon) x_{0}, \varepsilon y_{1}+(1-\varepsilon) x_{0}\right) \leq \varepsilon d\left(x_{1}, y_{1}\right) .
$$

By the definition of $z_{\varepsilon}$ and the uniformity of $d$ on the line connecting $z_{\varepsilon}$ with $x_{\varepsilon}$ and $y_{\varepsilon}$, we have

$$
d\left(x_{\varepsilon}, y_{\varepsilon}\right)=\varepsilon^{-1} d\left(x_{\varepsilon}, z_{\varepsilon}\right) \leq d\left(x_{1}, y_{1}\right) .
$$

Upon taking the limit $\varepsilon \rightarrow 0$ we therefore arrive at

$$
d\left(x_{0}, y_{0}\right) \leq d\left(x_{1}, y_{1}\right)
$$

and the other inequality direction is then clear by symmetry, so that $d$ is indeed translation invariant.

Now $d$ can be uniquely extended to a translation-invariant metric on the affine hull of $X$. Assuming $0 \in X$ without loss of generality, this affine hull equals the linear hull, and then the translation-invariant metric on $\operatorname{lin}(X)$ comes from a norm. If necessary, this norm can be extended from the subspace $\operatorname{lin}(X)$ to all of $E$. 
Now we have assembled all the ingredients for our main theorem:

Theorem 9. Every convex-like structure is affinely and isometrically isomorphic to a closed convex subset of a Banach space.

Proof. Since the inequality (6) is an instance of the metric compatibility axiom $(\gamma .4)$ this is a direct consequence of Corollary 6 and Lemma 8 and the fact that every norm space embeds into its completion, which is a Banach space. Closedness then follows from the requirement that a convex-like structure is assumed to be complete.

Remark 10. We have not used the completeness of $X$ in the derivation of Corollary 6 or Lemma (8). So if we would remove this hypothesis from the axioms, then we would get that (not necessarily complete) convex-like structures are precisely the convex subsets of normed spaces.

Remark 11. Given the axioms in Definition 1, Brown's original first metric compatibility condition

"There is a constant $C$ such that for all $x_{1}, \ldots, x_{n} \in X$,

$$
d\left(\gamma_{\mu}\left(x_{1}, \ldots, x_{n}\right), \gamma_{\tilde{\mu}}\left(x_{1}, \ldots, x_{n}\right)\right) \leq C \sum_{i=1}^{n}|\mu(i)-\tilde{\mu}(i)|, "
$$

holds if and only if $X$ is bounded (as a metric space).

Proof. By Theorem 9, we can take $X$ to be a closed convex subset of a Banach space, with the metric $d$ induced by the norm. Brown's condition then just states that

$$
\left\|\sum_{i=1}^{n}(\mu(i)-\tilde{\mu}(i)) x_{i}\right\| \leq C \sum_{i=1}^{n}|\mu(i)-\tilde{\mu}(i)| .
$$

If $X$ is bounded, then we can set $C=\sup _{x \in X}\|x\|$, and the inequality holds. Conversely, we can use (17) to deduce the boundedness of $X$ : taking $n=2$ and $\mu(1)=\tilde{\mu}(2)=1$ gives

$$
d\left(x_{1}, x_{2}\right)=\left\|x_{1}-x_{2}\right\| \leq 2 C .
$$

The following corollary is a reformulation of our previous results. It provides a simple way to axiomatize (closed) convex subsets of Banach spaces.

Corollary 12. Let $\left(X,\left\{c c_{\lambda}\right\}, d\right)$ be a convex space in the sense of Definition 2 together with a (complete) metric $d$. It is a (closed) convex subset of a Banach space if and only if it satisfies the inequality

$$
d\left(c c_{\lambda}(y, x), c c_{\lambda}(z, x)\right) \leq \lambda d(y, z) \quad \forall x, y, z \in X, \lambda \in[0,1] .
$$

\section{REFERENCES}

[Br] N.P. Brown, Topological Dynamical Systems Associated to $\mathrm{II}_{1}$-factors, Adv. Math. 227 (2011), 1665-1699. MR2799809

[Fr] T. Fritz, Convex Spaces I: Definition and Examples, Preprint, arXiv:0903.5522.

[Gu] S.P. Gudder, Convexity and Mixtures, SIAM Review 19, 2 (1977) 221-240. MR.0433327 (55:6304)

[Mo] P. Mongin, A note on mixture sets in decision theory, Decisions in Economics and Finance 24 (2001), 59-69. MR.1832228 (2002b:91030) 
[PR] D. Pumplün and H. Röhrl, Banach spaces and totally convex spaces. I, Comm. Algebra 12 (1984) 953-1019. MR735910 (86a:46094)

[Se] Z. Semadeni, Monads and their Eilenberg-Moore algebras in functional analysis, Queen's Papers in Pure and Applied Mathematics, No. 33 (1973).

[St] M.H. Stone, Postulates of barycentric calculus, Ann. Mat. Pura. Appl. 4, 29 (1949) 25-30. MR0036014 (12:45h)

Institut de Mathématiques, University of Neuchâtel, Rue Emile-Argand 11, CH-2000 NEUChÂTEL, SWITZERLAND

E-mail address: valerio.capraro@unine.ch

Institut de Ciències Fotòniques, Mediterranean Technology Park, 08860 CastelldeFELS (BARCElona), Spain

E-mail address: tobias.fritz@icfo.es 